\title{
STI-BT: A Scalable Transactional Index
}

\author{
Nuno Diegues and Paolo Romano \\ INESC-ID / Instituto Superior Técnico, Universidade de Lisboa, Portugal
}

\begin{abstract}
In this article we present STI-BT, a highly scalable, transactional index for Distributed Key-Value (DKV) stores. STI-BT is organized as a distributed B+Tree and adopts an innovative design that allows to achieve high efficiency in large-scale, elastic DKV stores. We have implemented STI-BT on top of a mainstream open-source DKV store and deployed it on a public cloud infra-structure. Our extensive experimental study reveals the efficiency of our solution with demonstrable scalability in a cluster of 100 commodity machines, and speed ups with respect to state of the art solutions of up to $5.4 \mathrm{x}$.
\end{abstract}

\section{INTRODUCTION}

The ever growing need for computational power and storage capacity has fostered research of alternative solutions to classic monolithic relational databases. In this context, Distributed Key-Value (DKV) stores (such as Dynamo [1]) became quite popular due to their scalability, fault-tolerance and elasticity. On the down side, developing applications using these DKV stores is far from trivial, due to two main factors: the adoption of weak consistency models and of simplistic primitives to access data.

Concerning data consistency, the inherent complexity of building applications on top of weakly consistent systems has motivated a flurry of works offering strongly consistent transactional semantics in large scale platforms [2]-[7].

On the other hand, the lack of indexing support for nonprimary data attributes forces programmers to either implement ad-hoc indexing strategies at the application level, or to rely on asynchronous indexing solutions [8], [9]. Neither approach is desirable, as the former is costly and error-prone, whereas the latter ensures only weak consistency.

In this paper we tackle this issue by introducing STI-BT, a transactional index designed to match the scalability and elasticity requirements of DKV stores. STI-BT is organized as a distributed $\mathrm{B}^{+}$Tree and adopts a unique design that leverages on the following mechanisms in a synergic way:

$\triangleright$ Data placement and data-driven transaction migration: a unique feature of STI-BT is that accesses to any indexed data item require normally only one remote access, regardless of the size of the index and of the number of machines in the system. This is achieved via the exploitation of data placement and transaction migration techniques, which are jointly leveraged to maximize data locality. STI-BT partitions the index into sub-trees that are distributed to different machines via lightweight data placement techniques based on custom consistent hashing [10] schemes. Transaction migration is used to relocate the execution of an operation to a remote machine when the data required is not available locally. This results in a drastic minimization of communication, enhancing the efficiency and scalability of the index.

$\triangleright$ Hybrid replication: STI-BT adapts the number of copies of the tree nodes according to their depth in the tree: top levels are fully replicated, whereas lower levels are partially replicated over a small set of machines. This brings a number of advantages. The nodes in the top of a $\mathrm{B}^{+}$Tree, despite representing a minimum fraction of the tree, account for a large part of the read accesses. By fully replicating them, STI-BT avoids the load unbalance that would otherwise occur if they were replicated only in a small set of machines. Also, top level nodes have the lowest probability of being updated, which makes the cost of maintaining them fully replicated negligible even in large clusters. Conversely, using partial replication for the lower levels (the majority of the data) ensures fault-tolerance, maximizes efficiency of storage, and keeps the cost of updating the index bounded at any scale of the system.

$\triangleright$ Elastic scaling: The ability to adjust the resources employed to the actual demand is one of the most attractive features of DKV stores. For this reason, STI-BT was tailored to ensure efficiency in the presence of shifts of the platform's scale. It reacts to changes by autonomously adjusting the boundaries of the fully replicated part, with the intent of minimizing it, and redistributing the index across the cluster.

$\triangleright$ Concurrency enhancing mechanisms: STI-BT combines a number of mechanisms to minimize data contention. STIBT is built on top of GMU [3], a recent distributed multiversioning scheme that executes read-only transactions in a wait-free fashion, hence allowing for executing lookup operations highly efficiently. To maximize scalability also in conflict-prone workloads, we used algorithms to navigate and manipulate the index that exploit concurrency-enhancing programming abstractions [11], [12] and drastically reduce the conflicts among concurrent index operations.

We have built STI-BT on top of Infinispan, a mainstream open-source transactional DKV store from Red Hat. We conducted an extensive experimental study, deploying STIBT in a large scale public cloud infrastructure (up to 100 machines) using benchmarks representative of OLTP workloads. The results highlight the high scalability of the 
proposed solution, which can achieve up to $5.4 \times$ speedups over state of the art solutions.

The rest of the paper is organized as follows. In Section II we discuss related work. Section III introduces background concepts and assumptions. Section IV overviews our solution, of which we provide a more detailed description through Sections V-VIII. Finally, we present our evaluation in Section IX, and conclude in Section X.

\section{RELATED WORK}

The development of $\mathrm{B}^{+}$Trees for indexing data constitutes a large body of work. One traditional usage targets centralized systems with persistent storage in disk [13], [14], whereas others have targeted distributed environments [15] (although without allowing atomic accesses to data items).

A Scalable BTree was proposed in [16] to index largescale data transactionally. Accesses to the tree are governed by Sinfonia [17], a distributed abstraction of shared memory with serializable transactions. However the main drawback is the focus on queries alone, for instance by fully replicating every tree node (except leafs). Thus, its performance lacks scalability under mixed workloads where the data is updated.

Another design of a distributed $\mathrm{B}^{+}$Tree was proposed in Minuet [18] (also on top of Sinfonia). Similarly to STIBT, Minuet also exploits multi-versioning to enhance concurrency between transactions. Yet, Minuet handles multiversioning externally to Sinfonia, by using a centralized snapshot identifier that is incremented whenever a read-only transaction requires a fresh snapshot. As we shall discuss in the next Section, our solution uses a scalable distributed multi-versioning scheme [3], which provides significant benefits for read-only transactions. Furthermore, Minuet distributes the tree nodes randomly across the Sinfonia cluster. In STI-BT, we exploit the structure of the tree to co-locate tree nodes and maximize data locality. Finally, we extend their usage of Dirty Reads (initially promulgated in [11]) to cope with Delayed Actions [12] for reduced concurrency conflicts in transactions.

The solution for large-scale data indexing presented in Global Index [19] also proposes to use tree structures, albeit in a different way. Every machine maintains the local data indexed in a local $\mathrm{B}^{+}$Tree and chooses only a subset of nodes to publish - publishing means adding the tree nodes to the Global Index, which is a BTree built using an peerto-peer overlay network called BATON [20]. The published nodes of some machine reflect an over-estimation of the data indexed at that machine, with the objective to reduce the frequency of expensive global synchronization upon updates of the index. The downside of this design is that, due to the inaccuracy of the published information, queries typically contact unnecessary machines, which can hinder performance. STI-BT's design, conversely, ensures that at most a remote machine is contacted to process an index operation, except for the rare case of concurrent rebalances affecting regions of the tree accessed by the operation. Another fundamental difference is that Global Index only ensures eventual consistency across replicas, whereas STIBT ensures strong consistency.

Other work has focused on indexing multi-dimensional data. The key idea of Global Index has been adapted to this purpose [21]: each machine indexes its local data in an R-tree to account for the multi-dimensions. Once again, the locality-efficient design contributions of STI-BT could be used in the distributed index that is built on top of these R-Trees. Other approaches to this topic rely also on distributed data-structures (such as the SkipTree [8]), on space-filling curves (as on Squid [22]), or on hyperspace hashing (as on HyperDex [23]). Many of these techniques have been developed in the context of Peer-to-Peer (P2P) network overlays - we refer to [24] for a recent survey on this topic. Traditionally, these systems were designed for environments with limited synchrony and high churn, as typical of large networks over the Internet. As such, they provide weak or no consistency guarantees at all, much less transaction abstractions that allow to atomically access and modify multiple data items. In contrast, STI-BT ensures strong consistency (without compromising scalability), and is designed to operate in clusters of cloud machines, which are more stable than typical $\mathrm{P} 2 \mathrm{P}$ systems.

\section{BACKGROUND AND ASSUMPTIONS}

Like other modern cloud data platforms [2], [17], Infinispan (our underlying DKV store) also supports transactional strong consistency. In particular, Infinispan integrates the GMU protocol [3], a strongly consistent transactional replication protocol that relies on a fully decentralized multiversioning scheme. Unlike classic solutions, GMU does not rely on a global logical clock, which may represent a contention point and impair scalability. Conversely, GMU determines version's visibility and transaction serialization order by means of vector clocks, which are updated genuinely (i.e., contacting only the machines involved in the execution of the transaction) and hence in a highly scalable fashion. Also, GMU never aborts read-only transactions and spares them from the costs of distributed validation, maximizing the efficiency of read-intensive workloads.

Concerning data placement, Infinispan relies on consistent hashing [10] to determine the placement of data (similarly to Dynamo [1], for instance). Consistent hashing is particularly attractive in large scale, elastic stores, as it avoids reliance on external lookup services, and allows to minimize the keys to be redistributed upon changes of the system's scale. However, deterministic random hash functions to map keys identifiers to machines lead to poor data locality [25], [26]. Hence, DKV stores using consistent hashing (like Infinispan) 
Table I: Description of terminology used through the paper.

\begin{tabular}{|c||c|}
\hline symbol & description \\
\hline \hline$\alpha$ & arity of a tree node \\
\hline $\mathcal{C}$ & cut-off level \\
\hline $\mathcal{M}$ & memory available in a machine \\
\hline $\mathcal{N}$ & number of machines in the cluster \\
\hline $\mathcal{K}$ & degree of partial replication \\
\hline
\end{tabular}

typically allow programmers to provide custom functions. As we will discuss in Section V, STI-BT relies heavily on novel, custom data placement strategies layered on top of consistent hashing to enhance data locality.

Finally, STI-BT relies on the structure of a $\mathrm{B}^{+}$Tree, where leaf nodes are connected to allow for in-order traversal and inner nodes do not contain values held by the index. We highlight Table I describing the symbols in our terminology.

\section{Design Rationale And OVERVIEW}

One of the main goals of STI-BT's design is to maximize data locality, i.e., to minimize the number of remote data accesses required to execute any index operation. This is a property of paramount importance not only to ensure efficiency, but also scalability. In fact, in solutions based on simplistic random data placement strategies, the probability of accessing data stored locally is inversely proportional to the number of machines. Hence, as the system scales, the network traffic generated by remote accesses grows accordingly, severely hindering scalability.

Data locality may be achieved using full replication, but that would constrain scalability from a twofold perspective. First, the cost of propagating updates (to all machines) grows with the size of the cluster. Second, it prevents scaling out the storage capacity of the system by adding machines.

We also highlight that partial replication techniques pose challenges to load balancing. Consider a simple approach in which each tree node is replicated across $\mathcal{K}=f+1$ machines, to tolerate up to $f$ faults in the cluster. Since the likelihood of accessing a tree node is inversely proportional to its depth in the tree, the machines maintaining the topmost tree nodes will receive a larger flow of remote data accesses, hence becoming bottlenecks of the system.

To cope with the issues mentioned above, STI-BT divides the $\mathrm{B}^{+}$Tree in two parts: the topmost $\mathcal{C}-1$ levels are fully replicated, whereas the bottom part, containing the $\mathcal{C}^{\text {th }}$ level downwards, is partially replicated. Here, $\mathcal{C}$ represents the cut-off level, i.e., the depth level of the tree where the nodes are no longer fully replicated. As we will discuss, $\mathcal{C}$ is a dynamic value, which is adjusted when the scale of the platform is altered. Hence, $\mathcal{C}$ is stored in a fully replicated key of the underlying key-value store, which ensures that its value can be known by any machine with transactional consistency. In addition to this, the bottom part of the tree is organized in co-located sub-trees. In Fig. 1 we can see the index distributed among 3 machines and the cut-off (we use $\mathcal{K}=1$ in the example).

The main advantage of this design is that, traversing down the tree at any given machine can, at most, incur in one remote access to another machine. If a traversal at machine $M_{1}$ reaches level $\mathcal{C}$, and requires data replicated at machine $M_{3}$, we take advantage of the co-location within each sub-tree to forward the execution flow of the transaction from $M_{1}$ to $M_{3}$. The $\mathrm{B}^{+}$Tree supports range searches by traversing down to the leaf holding the initial value of the interval of the search, and then following the pointers to the next leaf. Hence, we try to replicate neighbour sub-trees in the same machine, which also helps to minimize the communication required for range searches. This localityaware design results in a uniform load of the machines if the popularity of accesses is uniform across the indexed data.

STI-BT integrates also a set of mechanisms to minimize the likelihood of contention among concurrent operations on the index. The key idea is to address the two possible sources of contention in a $\mathrm{B}^{+}$Tree- structural changes of the tree topology and modifications of the node's contents - by exploiting the commutativity of the various operations supported by the index. This allows achieving high efficiency even in challenging update-intensive scenarios.

Another innovative algorithmic aspect of STI-BT is the management of the cut-off level $\mathcal{C}$. This is governed by two contradicting forces: (1) we aim to maintain the fully replicated part as small as possible, to ensure that rebalances are less likely to update inner nodes that are fully replicated; and (2) we need the cut-off level to be deep enough so that it contains enough tree nodes at that level to serve as sub-tree roots to load-balance between all the machines in the cluster. Based on these considerations, STI-BT reacts to the elastic scaling of the underlying DKV by adapting the number of fully replicated nodes in the distributed $\mathrm{B}^{+}$Tree.

\section{Maximizing Data Locality}

We now discuss in more detail how to achieve the colocation and forwarding of execution flow. We begin with the example in Fig. 1 with the flow of two index operations, one in $M_{1}$ and the other in $M_{3}$, each corresponding to a transaction being processed at each machine.

The transaction at $M_{1}$ accesses the index to obtain a data element in the sub-tree $B$. This sub-tree is stored at $M_{2}$, for which reason the traversal cannot be processed only with the data stored at $M_{1}$. To minimize communication, when the traversal reaches the level of depth $\mathcal{C}$ we move the request to $M_{2}$ and continue the traversal there. The design of STIBTensures that this can happen only once while traversing 


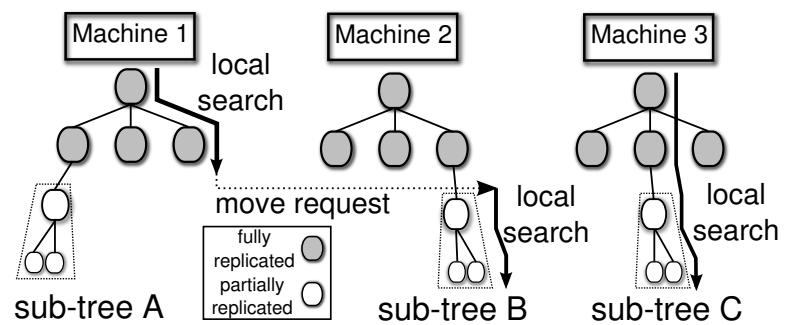

Figure 1: Example of communication flow in STI-BT.

the tree, which is optimal except when the request can be processed only with local data (as for the transaction at $M_{3}$ ).

Underlying this solution is the ability to co-locate data. For this, we still rely on the underlying consistent hashing from the DKV store, but extend it by means of a custom hashing scheme. To this end, we encode two parts in the identifier of each key $k$ (that maps to a tree node), i.e., $k=\left\langle k_{u}, k_{c l}\right\rangle:$ a unique identifier $\left(k_{u}\right)$, which identifies the node of the $\mathrm{B}^{+}$Tree, and a co-locality identifier $\left(k_{c l}\right)$, which is used to ensure co-location of different unique identifiers. STI-BT hashes only $k_{c l}$ when performing machines lookup for a key $k$, and uses $k_{u}$ when conducting local queries within a given machine. As a result, two different keys, sharing the co-location identifier $k_{c l}$, are hashed to the same machine. We exploit this by assigning the same $k_{c l}$ to all keys used to maintain the contents of a given sub-tree, which results in co-locating its tree nodes on a set of $\mathcal{K}$ machines determined via consistent hashing.

We also exploit the underlying DKV store's consistent hashing to govern the execution flow of transactions. To better present this idea, we shall rely on Algorithm 1. For simplicity, we omit the management of the DKV store when possible. Also, for the moment it suffices to consider that each tree node is mapped into a single key/value in the DKV store - we extend that in Section VII. Finally, we use a generic function portraying the role of operations accessing the tree (such as an insertion or range query), with focus on the common part of traversing down the tree.

We begin by considering a traversal in machine $M_{1}$ at a given tree node, as shown by the generic function in line 6 . The traversal goes down the tree nodes as long as those data items are fully replicated (verified through the metadata of the key that allows to access the tree node). When the next child tree node to traverse has a key that is partially replicated, one of two things can happen: (1) the key is locally replicated (the condition in line 10 is true), so the sub-tree is owned by $M_{1}$ and the operation is finished locally by calling function LOCALACCESS; or (2) the sub-tree is stored elsewhere, and so we decide to move the flow of execution (line 13 onwards). In the latter case, which can only occur at depth level $\mathcal{C}$ in our STI-BT, we create a task with the arguments of the access being performed in the

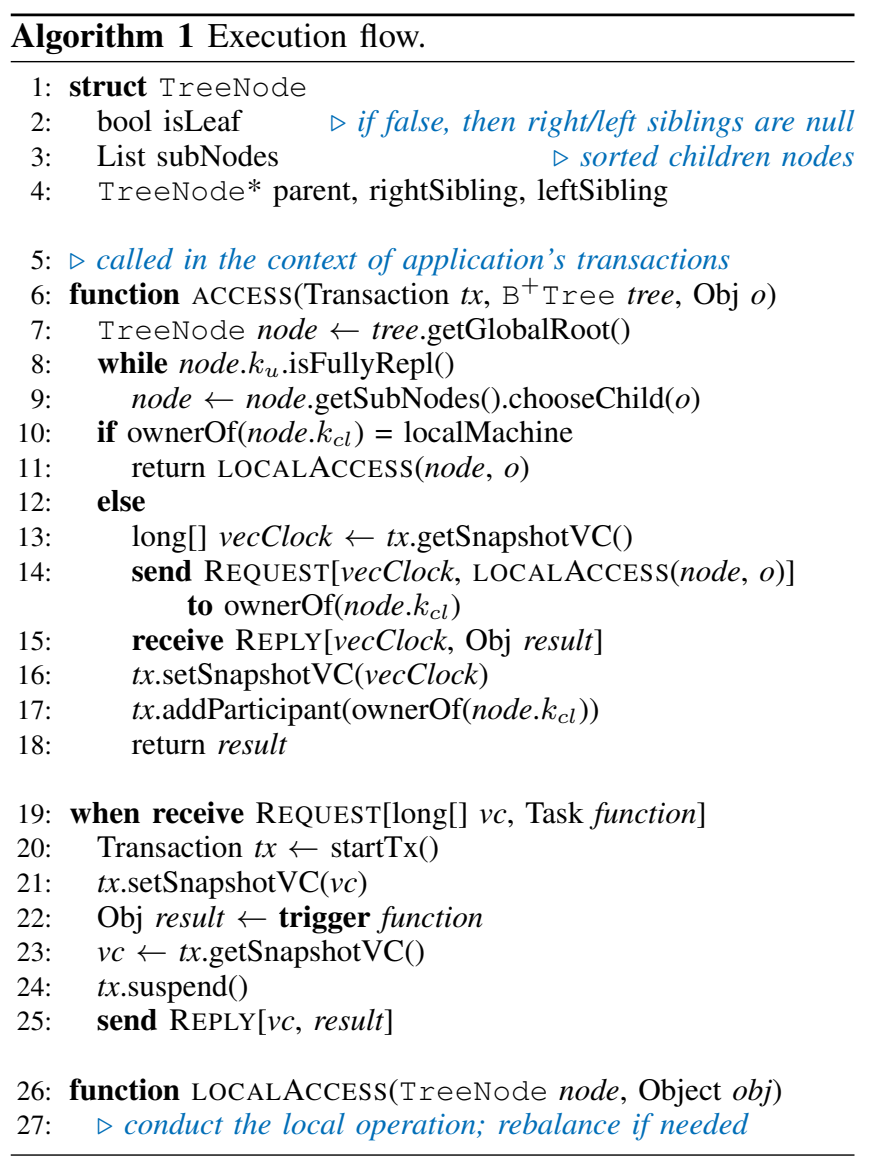

tree. We then use the consistent hashing function on the $k_{c l}$ identifier of the child's key to obtain the set of machines that replicate that data, and send the task to a random node in that set, which in our example is $M_{2}$ (line 14).

In fact, due to the transactional context under which these executions occur, we must send additional meta-data: the transaction that is executing at $M_{1}$ has necessarily performed some reads that restrict the snapshot of data that is visible to the transaction. This information is encoded via a vector clock in GMU (see Section III). Thus we retrieve the current transaction's vector clock (line 13) and send it to $M_{2}$, which answers back with a possibly updated vector clock reflecting any additional reads executed during execution at $M_{2}$. In practice, $M_{2}$ starts a new transaction that is forced to observe the snapshot used by transaction at $M_{1}$, thus guaranteeing consistency. No further meta-data is required at $M_{2}$ from $M_{1}$ : namely, we avoid moving the buffered write-set along with the transaction. This is because, before moving to $M_{2}$, the traversal at $M_{1}$ would only have read tree nodes and necessarily not written to any. On top of this, the remote execution will only read contents of the $\mathrm{B}^{+}$Tree (and not other data in the store), thus avoiding the 
possibility of a read-after-write. An exception to this occurs when multiple queries are invoked over the same tree in the course of a single transaction; in the event that these repeated invocations access the same parts of the tree, they will thus contact some previously contacted machines (say $M)$. In such case, we note that the part of the write-set that is relevant is already available at $M$, because it was created there during the previous remote execution(s).

After the execution returns to the origin machine of the transaction (for instance $M_{1}$ ), the transactional context is also updated (in line 17) to contain a reference to the transaction that was issued at a remote machine (for instance $M_{2}$ ). This remote transaction was suspended before the execution flow returned to the origin machine, and is thus pending a final decision. We extended the underlying DKV store to consider these additional participants in the distributed commit procedure. This means that $M_{2}$ will be seen as a participant to the distributed transaction coordinated by $M_{1}$, just as if $M_{1}$ had invoked some remote accesses to $M_{2}$.

Finally, we abstracted away the details of the usual implementation of $\mathrm{a} \mathrm{B}^{+}$Tree in the generic function LOCALACCESS. Note that modifications, such as insertion or removal, may require rebalances due to splits or merges. In such case, the rebalance operation goes back up and modifies the inner nodes as required. The likelihood of changing an inner node is proportional to its depth. Thus, it is normally unlikely that a rebalance reaches the top part of our STI-BT, which is fully replicated and incurs larger update costs.

\section{LoAd BALANCIng Sub-TREes}

The algorithm described so far took advantage of the existence of sub-trees with co-located data in the DKV store. Due to that design, a machine replicating more sub-trees ends up receiving more requests from remote nodes. On top of this, memory constraints may also apply (we assume that each machine has limited $\mathcal{M}$ memory capacity). For these reasons, STI-BT integrates a load balancing scheme aimed to homogenize resource consumption across machines.

As the $\mathrm{B}^{+}$Tree changes, rebalances occur and inner tree nodes are changed. In particular, at the $\mathcal{C}$ depth level, inner nodes may also change - we call the nodes at this level sub-tree roots because each one is a root of a sub-tree whose contents are all co-located. Conceptually, each machine has a list of sub-tree roots that is used to reason on the data of STI-BT that is stored there. We ensure that these lists (one per machine, per STI-BT) have balanced lengths in order to balance consumption of memory and processor (assuming that the popularity of indexed data is uniform).

To cope with load-balancing, each machine periodically triggers a routine to assess the number of sub-trees it currently owns. This procedure is executed under a transaction and it is re-attempted in case the transaction aborts due to a concurrency conflict.

The procedure, triggered at a given machine $M_{i}$, starts by assessing how many sub-trees the machine is responsible for. For this, it uses an array of all lists of sub-tree roots - one list per machine. It then computes the average size for all machines to assess the balance of the tree. We use a small tolerance value $\delta$ to avoid repeated migration of sub-trees between the same machines. If $M_{i}$ does not have an excess of sub-trees with respect to the average, then the procedure concludes. Otherwise, it means we can enhance the load-balancing by having $M_{i}$ offer some of its sub-trees to under-loaded machines: we change the keys of the tree nodes (being migrated) to the new $k_{c l}$ (that maps to a new machine, according to the consistent hashing).

So far we assumed for simplicity that a single, coarsegrained transaction encapsulated the whole migration procedure. Instead, for instance, it is possible to remove a root from a list of sub-tree roots and insert it in the other list in different transactions, because only the machine who owns a given root is responsible to migrate it else where - no two machines will race to migrate a given sub-tree. Hence, when changing the tree nodes to replicate them elsewhere, one can use a transaction to encapsulate the changes of each tree node. This minimizes the likelihood of conflicts and, in such events, the work to be repeated. The only cost of this optimization is that concurrent data accesses may have to traverse a partially collocated subtree. Consequently, in such rare event, STI-BT performs two remote executions when traversing down the tree.

\section{Minimizing Data Contention}

In order to minimize data contention among index operations, STI-BT relies on mechanisms aimed to avoid structural conflicts - i.e., allow traversals of the tree to be executed in parallel with tree rebalances, and to allow intranode concurrency - i.e., concurrent updates of different key/values in a tree node. To implement these mechanisms, we leverage on two programming abstractions proposed to enhance concurrency in transactional systems: Dirty Reads [11] and Delayed Actions [12]. Dirty Reads instruct the underlying concurrency control to avoid validating a read operation executed by the transaction. Delayed Actions allow for postponing the execution of conflict-prone code portions until the transaction's commit phase, where they can be executed in a sequential and conflict-free fashion.

STI-BT uses Dirty Reads when traversing down inner nodes of the $\mathrm{B}^{+}$Tree, and when navigating horizontally through the elements of a node. This allows for exploiting the commutativity among (update) operations that target different data items [27], allowing them to be successfully executed in parallel and avoiding unnecessary aborts (that would be triggered if plain reads were used). This technique 
brings additional benefits beyond minimizing data contention. Since the topmost part of STI-BT is fully replicated, if it did not use Dirty Reads to access fully replicated tree nodes, committing an update transaction would demand involving all machines in the cluster (to ensure consensus in validating the accesses to such nodes). The usage of Dirty Reads to access fully replicated nodes allows removing the corresponding keys from the transaction's read-set, significantly reducing network communication and the cost of maintaining the topmost part fully replicated.

Delayed Actions are used to avoid contention hotspots associated with the manipulation of the counters that maintain the number of elements stored by each tree node. Whenever an element is inserted to/removed from a node, the corresponding counter needs to be updated within the same transaction, and can become a contention point in conflict-prone workloads. To avoid this issue, we read this counter (to determine whether it is necessary to merge or split the node) using Dirty Reads, and update its value using a Delayed Action. As the latter takes place at commit time in an atomic step, this prevents that the update of a node's counter can ever cause the abort of the encompassing transaction. On the down side, this approach can lead to "missed" concurrent updates to a node's counter. This does not affect correctness but can cause an unbalance of the tree, which we detect and fix using a background thread in each machine that periodically checks the local sub-trees and rebalances them, if necessary. A detailed description of STIBT's concurrency mechanisms has to be omitted for space constraints, but can be found in our technical report [28].

\section{ELASTIC SCALING}

In cloud environments, the provisioning process of a DKV store is typically governed by an autonomic manager, which takes into account a number of factors (e.g., current load, energy consumption, utilization of computational and storage resources) and aims to ensure Quality-of-Service levels while minimizing costs [29], [30]. Regardless of the reasons that may determine a change in the machines of the cluster, STI-BT reacts by autonomously reconfiguring its structure (in particular, its cut-off level $\mathcal{C}$ ) to ensure optimal efficiency at any scale. If the cluster size changes, it is possible that the current $\mathcal{C}$ is not deep enough to contain enough sub-tree roots (at least one per machine). Conversely, upon a scale down, the sub-trees may exceed the actual need: this is also undesirable, as the shallower the cut-off, the less likely it is for an update to affect the fully replicated part.

Before presenting the details of the algorithm used to reconfigure $\mathcal{C}$, we first introduce an example to explain the high-level idea. Consider $\mathcal{C}=2$ and $\alpha=4$; then we have $\alpha^{\mathcal{C}}=16$ sub-trees available (assuming $\mathcal{K}=1$ ). Suppose that the cluster scales up and brings in a $17^{\text {th }}$ machine; then we cannot assign a sub-tree to the joining machine with the

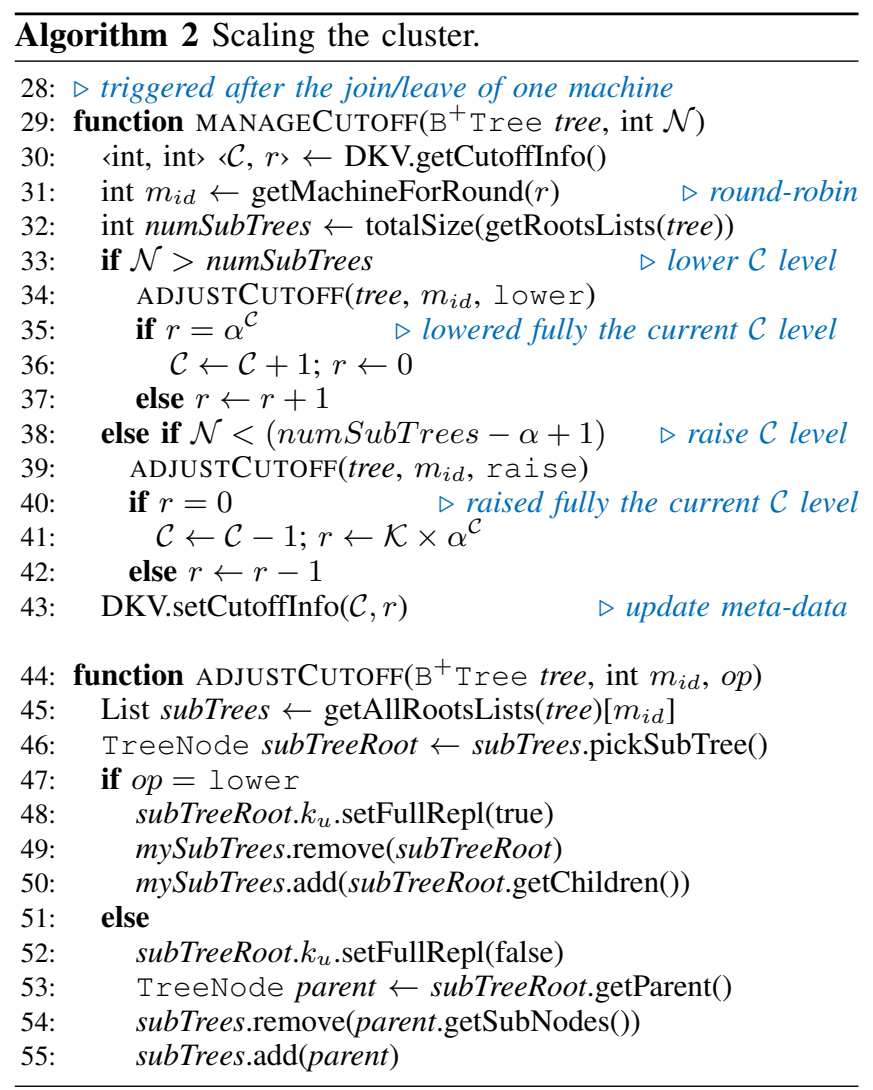

current $\mathcal{C}$. This motivates for deepening $\mathcal{C}$ (i.e., increment it) to create further sub-trees. Hence, by fully replicating $\alpha^{\mathcal{C}+1}$ inner nodes, we obtain $\alpha^{\mathcal{C}+1}=64$ total sub-trees. Yet, we do not need to be so aggressive: in fact, we do not need so many new sub-trees as we only brought in one new machine. The penalty here is that we are increasing considerably (and unnecessarily) the fully replicated part of the tree in a situation where we only acquired little new resources. By considering only one sub-tree root node in the fully replicated part of the index, we can turn its $\alpha$ children into new sub-tree roots, which can be migrated to new machines joining the DKV store. Hence, we adapt $\mathcal{C}$ using a finer-grained, more efficient strategy: we fully replicate the minimum sub-tree root nodes at the current $\mathcal{C}$ to create as many additional roots as the joining machines.

Algorithm 2 describes this procedure, which is triggered whenever a change of the DKV's scale is detected. For ease of presentation, the pseudo-code considers that the cluster size increases (or decreases) one machine at a time. As explained above, $\mathcal{C}$ needs to be adapted only if STI-BT has an insufficient (line 33) or excessive (line 38) number of sub-trees with respect to the new cluster size $\mathcal{N}$.

To manage the cut-off, we maintain some meta-data in 
the DKV store to ensure its coherency across machines. This meta-data is used to decide which sub-tree root will be moved to/from the fully replicated part (in lines 34 and 39). We use a round-robin strategy to pick a sub-tree from a different machine each time this procedure is executed, and use meta-data $r$ to keep track of the current round - the rounds count how many sub-trees of the current $\mathcal{C}$ have been lowered to $\mathcal{C}+1$. Hence why we only update $\mathcal{C}$ sometimes: $r$ consecutive growths (or shrinks) must occur before the full level is considered changed and the cut-off is changed.

The function ADJUSTCUTOFF is responsible for applying the cut-off change in the area of the tree corresponding to one sub-tree. When the objective is to lower the cut-off (due to the scale of the cluster increasing), this entails two things: (1) to make the current sub-tree root fully replicated, conceptually moving the cut-off to the next level in that part of the tree (line 48); and (2) to update the list of sub-tree roots that is used for load-balancing (lines 49-50). Lines 5255 conduct a symmetric raise procedure. These nodes belong to the top part of the tree and, since we use Dirty Reads to avoid validating reads issued on inner nodes, it is very unlikely for this procedure to incur any conflict.

Finally, we assess the impact of $\mathcal{C}$ on memory efficiency. Since the nodes above $\mathcal{C}$ are fully replicated, the more machines there are, the larger the portion of memory each one has to allocate to hold the fully replicated nodes. This is an additional motivation for minimizing $\mathcal{C}$. We evaluate the memory capacity $T C$ of STI-BT on a cluster of size $\mathcal{N}$ : The equation above subtracts $F R$ fully replicated nodes

$$
T C=\mathcal{N} \times(\mathcal{M}-\alpha \times F R) \quad \text { where } F R=\sum_{i=0}^{\mathcal{C}-1} \alpha^{i}=\frac{\alpha^{\mathcal{C}}-1}{\alpha-1}
$$

(each holding $\alpha$ keys of the DKV store) from the capacity of each machine. We can then evaluate the memory efficiency of STI-BT, noted $\eta$, as the ratio of its actual capacity to that of an ideal system whose total capacity scales perfectly with the number of machines (i.e., $T C=\mathcal{N} \times \mathcal{M}$ ): This

$$
\eta=1-\frac{\alpha(\mathcal{N}-1)}{(\alpha-1) \mathcal{M}}
$$

analysis highlights the efficiency of STI-BT in large scale deployments (containing hundreds or thousands of servers). In fact, even in such scenarios, it is realistic to assume that the number of keys held by each machine is much larger than the number of machines (i.e., $\mathcal{M} \gg \mathcal{N}$ ), yielding a memory efficiency very close to 1 for any, non-minimal value of $\alpha$.

\section{EXPERIMENTAL EVALUATION}

We developed a prototype of STI-BT on top of Infinispan, a popular in-memory transactional DKV developed by Red Hat. Each experiment uses $\mathcal{K}=2$ for fault-tolerance, and the reported results represent the average over 10 runs. We use geometric mean for averages over normalized results. All tests were executed using $\alpha=25$, unless specified otherwise. We conducted our tests on FutureGrid, a large scale public cloud infrastructure, from which we acquired a pool of up to 100 virtual machines equipped with 2 physical cores, 4GB of RAM and interconnected via InfiniBand. We also present experiments using a single many-core machine with 48 AMD Opteron cores at $2.1 \mathrm{Ghz}$ and 128GB RAM.

\section{A. YCSB Workloads}

YCSB [31] is a popular benchmark for DKV stores, whose workloads comprehend single key operations (read, insert and modify) as well as range scans and read-modifywrite operations, emulating data access patterns of real applications (typically skewed). We used strong scaling by always loading the index with $10 \mathrm{~GB}$ of data drawn from a uniform distribution (our experiments evidenced that larger data-sets had no impact on the results). Finally we also scale the number of clients with the number of machines running the key-value store (co-located processes). To help understand the benefits of STI-BT, we created several $\mathrm{B}^{+}$Tree variants:

$\triangleright$ Baseline: $\mathrm{a}^{+}$Tree built on top of Infinispan without any of the contributions mentioned in this paper.

$\triangleright$ Sub-Trees: this version enhances Baseline by exploiting sub-tree co-location and execution migration. Hence, transactions perform fewer remote accesses. The topmost part, however, is partially replicated. Thus, traversing the first tree nodes results in remote accesses with a high likelihood.

$\triangleright$ Dirty: this version adds Dirty Reads to the Baseline, by exploiting them when accessing inner nodes. In this scheme, tree nodes are placed randomly, which causes a high number of remote accesses. The advantage of this variant is that update transactions (that require commit time validation) involve a smaller number of machines in the commit phase because Dirty Reads need not be validated.

$\triangleright$ TopFull: this version differs from the Baseline by fully replicating the topmost part of the tree (the nodes above $\mathcal{C})$. When traversing these nodes, this variant also uses Dirty Reads, to avoid contacting all machines (due to full replication) during transaction's validation. However, this variant is expected to reduce remote accesses only slightly, as all operations imply traversing partially replicated parts of the tree scattered using random placement.

We start by showing, in Fig. 2, experiments for each of YCSB's workloads using 60 machines, which corresponds to a medium scale deployment given the maximum size of our experimental test-bed. The results highlight the significant speed ups achieved by STI-BT over the Baseline (average throughput improvement of $13.5 \times$ ) regardless of the workload. By evaluating the relative improvement achieved by each variant, the plots allow us to analyze the relevance of each design contribution. In particular, we obtained the 


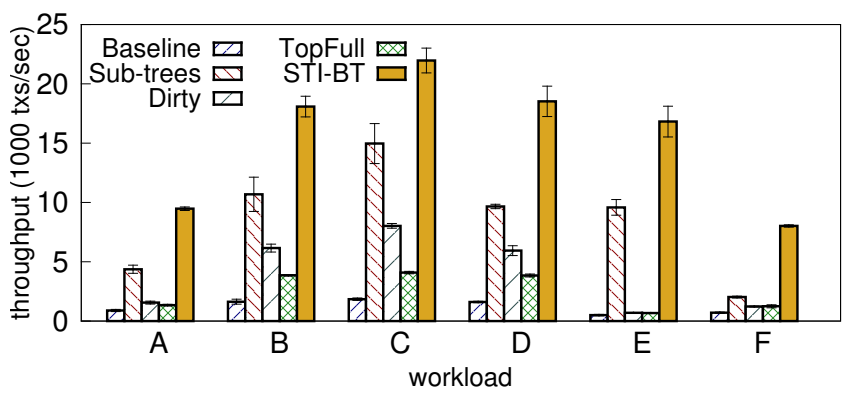

Figure 2: YCSB workloads with 60 machines.

following speedups over the Baseline: $6.6 \times$ for Sub-Trees; $2.5 \times$ for Dirty; and $1.9 \times$ for TopFull. Note that each contribution on its own never reaches more than half the throughput of STI-BT; this fact will be more evident when we look at individual workloads. In Table II, we show the number of remote data fetches per transaction, and the number of machines contacted per commit. For STI$\mathrm{BT}$, the remote operations represent only the migration of control flow (on average once per transaction) and rebalance operations that require updating nodes belonging to two subtrees that are not assigned to the same machine. Sub-Trees requires further remote accesses due to the topmost part not being fully replicated. Finally, the Dirty design does not reduce the remote accesses, and the TopFull variant reduces this number only marginally. Note that the Dirty variant reduces the machines contacted by a larger extent than TopFull because of the reduced read-set for validation.

We now look in more detail at Workload A in Fig. 3a, which shows the throughput of each variant as the platform's scale grows, highlighting that STI-BT scales until 100 machines almost linearly even in this challenging, update intensive workload. We also show the peak throughput using a 48-core machine, which does not ensure fault-tolerance (no replication/distribution overhead) for reference purposes of the absolute throughput. In this experiment we note that all other variants besides STI-BT are either not scalable, or achieve inferior performance. In particular, the Sub-Trees variant performs best among them, but its trend stagnates
Table II: Remote gets | machines contacted, relative to Fig.2.

\begin{tabular}{|c||c|c|c|c|c|c|}
\hline & A & B & C & D & E & F \\
\hline \hline Baseline & $15 \mid 19$ & $14 \mid 15$ & $10 \mid 9$ & $12 \mid 14$ & $32 \mid 29$ & $14 \mid 22$ \\
\hline Sub-Trees & $3 \mid 5$ & $3 \mid 5$ & $2 \mid 4$ & $2 \mid 4$ & $3 \mid 5$ & $2 \mid 4$ \\
\hline Dirty & $15 \mid 15$ & $14 \mid 12$ & $10 \mid 9$ & $12 \mid 12$ & $30 \mid 23$ & $14 \mid 15$ \\
\hline TopFull & $9 \mid 18$ & $8 \mid 11$ & $7 \mid 8$ & $8 \mid 12$ & $16 \mid 26$ & $11 \mid 19$ \\
\hline STI-BT & $0.2 \mid 2$ & $0.3 \mid 2$ & $0.1 \mid 2$ & $0.1 \mid 2$ & $1.4 \mid 3$ & $0.1 \mid 2$ \\
\hline
\end{tabular}

at large scale. This strengthens the relevance of combining the whole set of mechanisms included by STI-BT, as each one alone performs rather poorly. This is also confirmed in the Cumulative Distribution Function of the transactions' execution latencies (see Fig. 3c): while STI-BT processed $90 \%$ of the transactions in $6 \mathrm{~ms}$ (or less), this value is, respectively, $2 \times, 3 \times, 6 \times$ and $10 \times$ higher for Sub-Trees, Dirty, TopFull and Baseline.

Workload E, instead, requests $95 \%$ range scans and $5 \%$ insertions (see Fig. 3d). The results show that the gains in throughput and latency of STI-BT are even larger than for workload A (except for Sub-Trees). This workload is scanheavy, for which reason co-locating sub-trees is even more important, as it allows traversals at the leaf nodes to be conducted locally most of the times. Hence, in this workload, the Sub-Trees feature is clearly the most important, whereas the others are of no help. In fact, the latency CDF of the other variants has a longer tail due to traversals that take up to hundreds of milliseconds. Note that these scan requests are wrapped in read-only transactions that are abort-free.

\section{B. Fresh Read-Only Transactions}

The Minuet B-Tree [18] shares the design of the Dirty variant that we showed above, without any co-location or placement as the Sub-Trees and TopFull variants. Also Minuet relies on multi-versioning, but it adopts a fundamentally different approach to implementing it. STI-BT is layered on top of GMU, which relies on a scalable vector clock-based distributed timestamping mechanism, that avoids to contact any other machines than those maintaining data accessed by the transaction. Conversely, Minuet relies on a shared

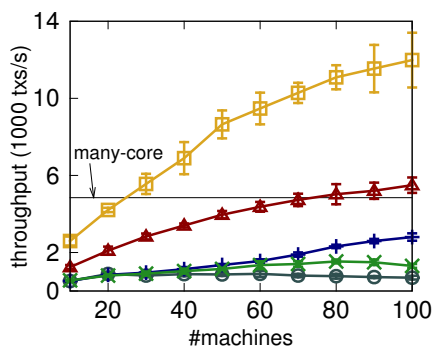

(a) Throughput in A.

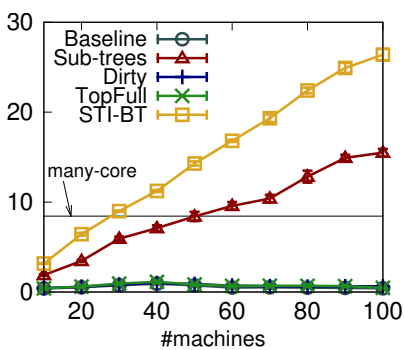

(b) Throughput in E.

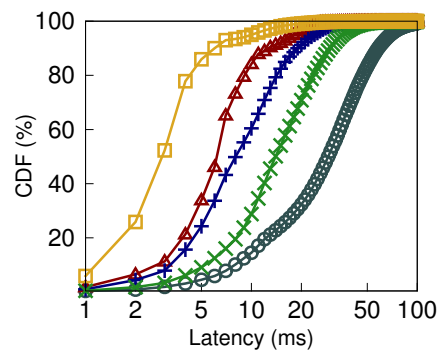

(c) Latency $\mathrm{CDF}$ in $\mathrm{A}$.

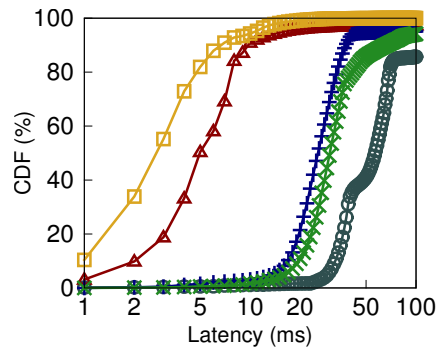

(d) Latency CDF in E.

Figure 3: Scalability and Latency CDF (at 60 machines) for YCSB's workloads A (heavy update) and E (short scans). 


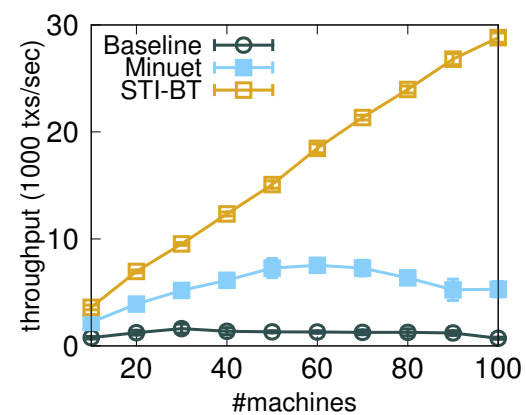

Figure 4: YCSB workload D.

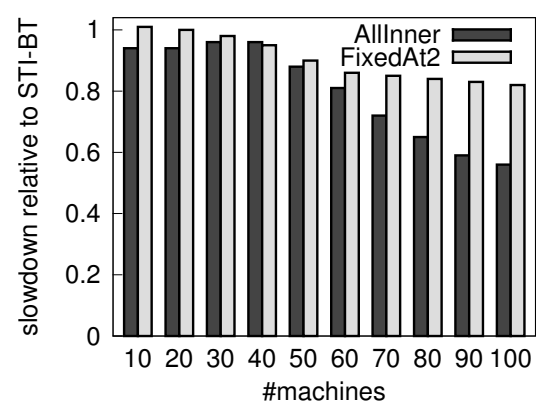

Figure 5: Varying cut-offs $(\mathcal{C})$.

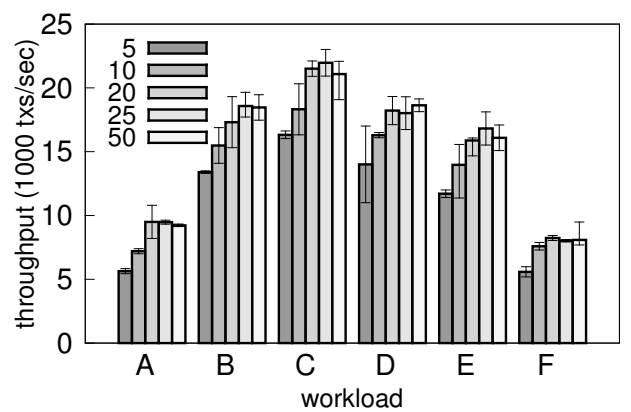

Figure 6: Varying arity $(\alpha)$ in YCSB. global clock (maintained by a single machine), which is incremented whenever a read-only transaction requests a fresh view of the data. As we will show, this centralized design hinders performance and compromises scalability.

In Fig. 4 we show detailed results obtained with workload $\mathrm{D}$, which mimics accesses to the latest available data (readdominated). As the source code of Minuet is not available, we emulated it by using a fully fledged STI-BT where read-only transactions first run an update transaction that increments a partially replicated key-value representing the shared clock. When that increment fails, we do not repeat the update transaction, and instead use the borrowing technique introduced in Minuet [18]. Note that the way in which we implemented Minuet is clearly favouring it, as it also benefits from the smart data placement of STI-BT (not used in Minuet). Regardless of that, this Minuet-like solution is clearly not scalable when faced with workloads that require fresh data. Conversely, STI-BT scales up almost to 30 thousand transactions per second with 100 machines while always providing fresh, consistent snapshots.

\section{Cut-off Adaptation and Tree Arity}

To assess the effects of adapting $\mathcal{C}$ as the system scale changes, we consider two alternative, static strategies: AllInner, which fully replicates all inner nodes (similarly to [16]), and FixedAt2, which places the cut-off at depth 2.

In Fig. 5 we show the slowdown of both strategies vs our adaptive mechanism, using Workload A. FixedAt2 performs similarly to STI-BT when the cluster size is small; but as more machines join, the sub-tree assignment becomes unbalanced, as some machines keep more trees than others. AllInner's performance is significantly lower, as rebalance operations are likely to modify fully replicated inner nodes, an inherently non-scalable operation.

In Fig. 6, we show the average throughput of STI-BT across all YCSB workloads while varying the tree arity. We can see that performance increases slightly as the arity increases, and eventually stagnates. This is due to the fact that low arity values lead to deeper trees, which cause a higher number of accesses to the underlying DKV. However, this effect becomes negligible as the arity increases. Overall, this shows that STI-BT ensures stable performance for non-minimal arity values (beyond 20, which motivated the settings of $\alpha$ for the other experiments presented).

\section{Conclusions}

In this paper we have presented STI-BT, a scalable solution to index data transactionally on a DKV store. STIBT allows overcoming one of the inherent, and most severe limitations of this emerging type of platforms: the lack of (efficient) transactional indexes for non-primary attributes. STI-BT ensures that the index is transactionally consistent with the data, sparing programmers from the complexity of asynchronous/weakly consistent indexing solutions. This is achieved without compromising the key strength points that have determined the success of DKV stores, namely scalability and elasticity.

STI-BT combines a number of innovative mechanisms aimed to i) maximize data locality, ii) achieve optimal efficiency at any scale, and iii) minimize data contention. We integrated STI-BT in a mainstream transactional DKV store, and conducted an extensive experimental study. Our results demonstrate its scalability and efficiency, by achieving linear scalability in a cluster of 100 commodity machines, and up to $5.4 \times$ speed-ups over state of the art solutions.

\section{ACKNOWLEDGMENT}

This work was supported by national funds through FCT (Fundação para a Ciência e Tecnologia) under project PEstOE/EEI/LA0021/2013 and by projects Cloud-TM (257784), specSTM (PTDC/EIA-EIA/122785/2010), and GreenTM (EXPL/EEI-ESS/0361/2013).

\section{REFERENCES}

[1] G. DeCandia et. al., "Dynamo: Amazon's Highly Available Key-value Store," in Proc. Symposium on Operating Systems Principles (SOSP), 2007, pp. 205-220.

[2] J. Corbett et al., "Spanner: Google's globally-distributed database," in Proc. Conference on Operating Systems Design and Implementation (OSDI), 2012, pp. 251-264. 
[3] S. Peluso, P. Ruivo, P. Romano, F. Quaglia, and L. Rodrigues, "When Scalability Meets Consistency: Genuine Multiversion Update-Serializable Partial Data Replication," in Proc. International Conference on Distributed Computing Systems (ICDCS), June 2012, pp. 455-465.

[4] S. Peluso, P. Romano, and F. Quaglia, "SCORe: A Scalable One-Copy Serializable Partial Replication Protocol," in Proc. Middleware, 2012, pp. 456-475.

[5] A. Turcu, B. Ravindran, and R. Palmieri, "Hyflow2: a high performance distributed transactional memory framework in scala," in Proc. on Principles and Practices of Programming on the Java Platform (PPPJ), 2013, pp. 79-88.

[6] M. S. Ardekani, P. Sutra, and M. Shapiro, "Non-Monotonic Snapshot Isolation: scalable and strong consistency for georeplicated transactional systems," in Proc. International Symposium on Reliable and Distributed Systems (SRDS), 2013, pp. 163-172.

[7] Y. Sovran, R. Power, M. K. Aguilera, and J. Li, "Transactional storage for geo-replicated systems," in Proc. Symposium on Operating Systems Principles (SOSP), 2011, pp. 385-400.

[8] S. Alaei, M. Ghodsi, and M. Toossi, "Skiptree: A new scalable distributed data structure on multidimensional data supporting range-queries," Computer Communications, vol. 33, no. 1, pp. 73-82, Jan. 2010.

[9] D. Logothetis and K. Yocum, "Ad-hoc data processing in the cloud," Journal Proc. VLDB Endowment, vol. 1, no. 2, pp. 1472-1475, Aug. 2008.

[10] D. Karger, E. Lehman, T. Leighton, R. Panigrahy, M. Levine, and D. Lewin, "Consistent hashing and random trees: distributed caching protocols for relieving hot spots on the World Wide Web," in Proc. Symposium on Theory of Computing (STOC), 1997, pp. 654-663.

[11] M. Herlihy, V. Luchangco, M. Moir, and W. N. Scherer, III, "Software Transactional Memory for Dynamic-sized Data Structures," in Proc. 22nd Symposium on Principles of Distributed Computing (PODC), 2003, pp. 92-101.

[12] N. Diegues and P. Romano, "Bumper: Sheltering Transactions from Conflicts," in Proc. International Symposium on Reliable and Distributed Systems (SRDS), 2013, pp. 185-194.

[13] P. L. Lehman and s. B. Yao, "Efficient locking for concurrent operations on B-trees," ACM Transactions on Database Systems, vol. 6, no. 4, pp. 650-670, Dec. 1981.

[14] G. Graefe, "A survey of B-tree locking techniques," $A C M$ Transactions on Database Systems, vol. 35, no. 3, pp. 1-26, Jul. 2010.

[15] J. MacCormick, N. Murphy, M. Najork, C. A. Thekkath, and L. Zhou, "Boxwood: abstractions as the foundation for storage infrastructure," in Proc. Conference on Symposium on Opearting Systems Design and Implementation (OSDI), 2004, pp. 8-24.

[16] M. K. Aguilera, W. Golab, and M. A. Shah, "A Practical Scalable Distributed B-tree," Journal Proc. VLDB Endowment, vol. 1, no. 1, pp. 598-609, Aug. 2008.
[17] M. K. Aguilera, A. Merchant, M. Shah, A. Veitch, and C. Karamanolis, "Sinfonia: A New Paradigm for Building Scalable Distributed Systems," in Proc. Symposium on Operating Systems Principles (SOSP), 2007, pp. 159-174.

[18] B. Sowell, W. Golab, and M. A. Shah, "Minuet: A Scalable Distributed Multiversion B-tree," Journal Proc. VLDB Endowment, vol. 5, no. 9, pp. 884-895, May 2012.

[19] S. Wu, D. Jiang, B. C. Ooi, and K.-L. Wu, "Efficient B-tree Based Indexing for Cloud Data Processing," Journal Proc. VLDB Endowment, vol. 3, no. 1-2, pp. 1207-1218, Sep. 2010.

[20] H. V. Jagadish, B. C. Ooi, and Q. H. Vu, "BATON: a balanced tree structure for peer-to-peer networks," in Proc. 31st International Conference on Very Large Data Bases (VLDB), 2005, pp. 661-672.

[21] J. Wang, S. Wu, H. Gao, J. Li, and B. C. Ooi, "Indexing multidimensional data in a cloud system," in Proc. International Conference on Management of Data (SIGMOD), 2010, pp. 591-602.

[22] C. Schmidt and M. Parashar, "Squid: Enabling search in DHT-based systems," Journal of Parallel and Distributed Computing, vol. 68, no. 7, pp. 962-975, 2008.

[23] R. Escriva, B. Wong, and E. G. Sirer, "HyperDex: A Distributed, Searchable Key-value Store," in Proc. Conference on Applications, Technologies, Architectures, and Protocols for Computer Communication (SIGCOMM), 2012, pp. 25-36.

[24] C. Zhang, W. Xiao, D. Tang, and J. Tang, "P2p-based multidimensional indexing methods: A survey," Journal of Systems and Software, vol. 84, no. 12, pp. 2348-2362, 2011.

[25] J. Paiva, P. Ruivo, P. Romano, and L. Rodrigues, "AutoPlacer: scalable self-tuning data placement in distributed key-value stores," in Proc. International Conference on Autonomic Computing (ICAC), 2013, pp. 119-131.

[26] A. Turcu, R. Palmieri, and B. Ravindran, "Automated data partitioning for highly scalable and strongly consistent transactions," in Proc. International Systems and Storage Conference (SYSTOR), 2014.

[27] J. Kim, R. Palmieri, and B. Ravindran, "Enhancing Concurrency in Distributed Transactional Memory through Commutativity," in Proc. Euro-Par, 2013, pp. 150-161.

[28] N. Diegues and P. Romano, "STI-BT: A scalable transactional index,” INESC-ID, Tech. Rep. 24, September 2013.

[29] S. Das, D. Agrawal, and A. El Abbadi, "ElasTraS: An elastic, scalable, and self-managing transactional database for the cloud," ACM Transactions on Database Systems, vol. 38, no. 1, pp. 1-45, Apr. 2013.

[30] D. Didona, P. Romano, S. Peluso, and F. Quaglia, "Transactional Auto Scaler: Elastic Scaling of In-memory Transactional Data Grids," in Proc. 9th International Conference on Autonomic Computing (ICAC), 2012, pp. 125-134.

[31] B. Cooper, A. Silberstein, E. Tam, R. Ramakrishnan, and R. Sears, "Benchmarking cloud serving systems with YCSB," in Proc. Symposium on Cloud Computing (SoCC), 2010, pp. $143-154$ 